\section{COMPLEJIDAD DINÁMICA EN \\ INTERNET COMO PLATAFORMA DE \\ INFORMACIÓN Y COMUNICACIÓN: \\ análisis filosófico desde la perspectiva de Ciencias de Diseño y el papel de la predicción}

Wenceslao J. González*

\begin{abstract}
RESUMEN
Debido al intenso desarrollo, cuantitativo y cualitativo, de Internet, se ha generado el problema de la complejidad dinámica en la Red. Su constante crecimiento como sistema abierto, donde proliferan nuevas expresiones - de contenido, funciones, etc.-, genera nuevas formas de complejidad, que son de suyo dinámicas. Para afrontar este problema, se realiza aquí un análisis filosófico desde la perspectiva de Ciencias de Diseño y el papel de la predicción, pero buscando superar el planteamiento de Herbert Simon. Porque el problema de la complejidad dinámica en Internet hay que afrontarlo desde la historicidad en los campos cognitivo, práctico y evaluativo.
\end{abstract}

Palabras clave: Crecimiento. Red. Artificial. Futuro. Historicidad.

\footnotetext{
* Catedrático de Lógica e Filosofía da Ciência na Universidade de la Coruña, Espanha. Académico da Academia Internacional de Filosofía da Ciência. Fellow del Center for Philosophy of Science de la Universidad de Pittsburgh, Estados Unidos. E-mail:wencglez@udc.es.
}

\section{INTRODUCCIÓN}

$\mathrm{H}$ oy Internet plantea un relevante problema filosófico: el análisis de su complejidad dinámica, que es clave para entender la situación actual y la proyección futura de la Red. Es una característica que aparece debido al intenso desarrollo, cuantitativo y cualitativo, de la Red, que genera un grado de complejidad creciente con los cambios a través del tiempo. Es una complejidad dinámica, porque acompaña al constante crecimiento de Internet como sistema abierto, universalmente accesible y versátil en sus cometidos, donde incide la actividad científica y el quehacer tecnológico, junto con un amplio conjunto de elementos contextuales (sociales, culturales, económicos, etc.). Pero es un tema que, en rigor, Winter y Ono (2015) no indagan de manera directa en sus perspectivas de futuro acerca de Internet.

Entre los rasgos de la complejidad dinámica de Internet están los siguientes: (i) epistemológicamente, está asociada a la constante novedad - de contenidos, funciones, aplicaciones, etc. - de Internet como plataforma multivariada, que inicialmente se centraba en la información y la comunicación. (ii) Metodológicamente, se combinan progresivamente en ella una serie de planos "la complejidad estructural, al menos epistemológica y ontológica facetas globalmente consideradas por (RESCHER, 1998, p. 8-16), y, desde otro ángulo, por (BISHOP, 2011)", con una creciente sofisticación en sus objetivos (muchos son genuinamente nuevos, no previstos en el proyecto inicial). (iii) Ontológicamente, inciden en ella los diversos niveles de la Red -micro, meso y macro-, en cuanto que atañe a los distintos tipos de diseños y sus cambios a través del tiempo. Así, se plantea tanto en términos específicos - mediante contribuciones científicas orientadas a objetivos concretos - como desde una perspectiva global (que incluye incremento del formato audiovisual y nuevos cometidos). (iv) Axiológicamente, no se puede caracterizar adecuadamente como "evolución", toda vez que tiene genuina historicidad la interacción a través del tiempo entre una vertiente interna y otra 
externa de la Red (una Historia de Internet, vista desde la web, por BERNERS-LEE, 1999).

Para afrontar el problema de la complejidad dinámica en Internet, se realiza aquí un análisis filosófico desde la perspectiva de Ciencias de Diseño y el papel de la predicción. A este respecto, se dan varios pasos: 1) Análisis de los principales factores dinámicos que, relacionados con la complejidad en la Red, intervienen en el caso de Internet. 2) Consideración de la complejidad dinámica desde el ángulo científico, a partir de las Ciencias de Diseño, buscando superar el planteamiento de Simon. 3) Ciencias actualmente existentes relacionadas con Internet y modalidades de investigación científica relacionadas con la Red.4) Papel de la predicción, que es clave para abordar la complejidad dinámica, en cuanto que anticipa el futuro posible. 5) Tarea de la historicidad para afrontar el problema de la complejidad dinámica en Internet. 6) Nuevas posibilidades filosóficometodológicas abiertas por el nuevo enfoque.

\section{FACTORES EN EL ANÁLISIS FILOSÓFICO DE LA COMPLEJIDAD DINÁMICA EN INTERNET}

Surge la complejidad dinámica a partir de los factores de origen. Así, en primer lugar, están los factores de la configuración de Internet, donde dos ángulos principales -el científico y el tecnológico - están estrechamente interrelacionados en la Red (algo que también sucede en Inteligencia Artificial (AUTOR, 2017), que le sirve con frecuencia de base a desarrollos de Internet). Pero la creatividad científica y la innovación tecnológica son conceptualmente distinguibles (en especial, en cuanto a la modalidad de racionalidad utilizada).

Después, en segundo término, se encuentran los factores de desarrollo, por vía de diversificación, según dos vertientes en cada uno de esos ángulos. (i) La vertiente interna incide en los objetivos, procesos y resultados de la actividad científica y en las metas, transformaciones operativas y productos del quehacer tecnológico. (ii) La vertiente externa se ocupa de la relación con el entorno (social, cultural, político, económico, ecológico, legal, etc.) de la actividad científica de la Red y del quehacer tecnológico de Internet. Esas dos vertientes -interna y externa - están en una relación bidireccional. No son meros "estratos" superpuestos o "corrientes" en paralelo: en la Red se plantean problemas entre los diseños el cometido primariamente interno - y el papel de los usuarios - la práctica de los agentes externos - que interactúan con los resultados de los diseños.

Ya en tercera instancia se aprecia que todo lo anterior -origen y desarrollo por vía de diversificación - está condicionado por la historicidad. Porque los factores de los ángulos científico y tecnológico y los propios de las dos vertientes -interna y externadependen de la variabilidad en el tiempo que no se puede concebir, en rigor, solo como genéricos "procesos" o como mera "evolución" (AUTOR, 2013a). En efecto, el tipo de dinámica en Internet introduce cambios profundos - no solo "horizontales" o ampliativos, sino también "verticales" o transversales - y, además, propicia modificaciones en varios niveles (micro, meso y macro). A este respecto, aquí se pone de relieve la historicidad asociada a Internet y se reflexiona preferentemente sobre el ángulo científico y la vertiente interna (HOOKER, 2011, p. 215), porque han recibido más atención el ángulo tecnológico (HANSETH; LYYTINEN, 2010, UHLIG, 2010) y la vertiente externa, que insiste en la dimensión histórica y social de Internet (FLORIDI, 2014).

Al hacer aquí el análisis filosófico en términos de Ciencia de Diseño, se busca superar la concepción de Simon (1996) sobre las Ciencias de lo Artificial en varias direcciones. 1) Se resalta que la complejidad dinámica es tan importante al menos como la complejidad estructural, mientras que Simon se centra primordialmente en la complejidad estructural, pues su interés primario es la "arquitectura de la complejidad" (SIMON, 1962). 2) Se destaca que los tipos de cambios en el campo científico-artificial, como es el caso de la Red, dependen de diseños que, con frecuencia, no se reducen a la mera "evolución". Porque el grado de amplitud, profundidad y diversidad de los cambios - que han sido muy intensos en las últimas décadas - solo se puede explicar desde la historicidad, que mira hacia una actividad en lugar de atender meramente a una conducta. Así, Internet ha revolucionado fines y medios de la comunicación y el intercambio de información: servidores de correo electrónico, YouTube, Facebook, Snapchat, Twitter, etc. 3) El papel de la His- 
toria en cualquier disciplina científico-artificial no es puramente contextual y como mero marco adaptativo de conductas (SIMON, 1998), sino que hay una dinámica interna de los avances realizados, de índole histórica propiamente dicha, donde intervienen diversos aspectos.

\section{ENFOQUE DE LA COMPLEJIDAD DINÁMICA DESDE EL ÁNGULO CiENTífico: Las Ciencias de Diseño}

Visto el problema de la complejidad dinámica en Internet desde el ángulo científico -que lleva al campo de las Ciencias de lo Artificial- y en vertiente interna, cabe apreciar al menos tres componentes principales: la epistemológica, la metodológica y la ontológica. Porque, para analizar la trayectoria de la Red y su posible avance futuro, hay que contar a) con un conocimiento - teórico y empírico - abierto a nuevas posibilidades, b) unos métodos que permitan la resolución de dificultades concretas mediante un número finito de pasos, y c) la existencia de nuevas expresiones ontológicas, como las propiedades emergentes. Cada una de ella requiere predicción con respecto al futuro, pues hay que conocer el futuro posible, tener métodos para abordarlo y caracterizar hechos nuevos.

Hace falta, además, una concepción de las Ciencias de Diseño más amplia que las Ciencias de lo Artificial de Simon (1986) y un enfoque de Internet más abarcante que el ofrecido por Graham (1999), para poder seguir esas direcciones epistemológica, metodológica y ontológica. Así, para superar las limitaciones de estos pensadores, se requiere una visión más elaborada de la racionalidad y la predicción en Ciencias de Diseño (AUTOR, 2008a). A este respecto, la trayectoria de la complejidad dinámica de Internet y su posible avance futuro requieren nuevos planteamientos, para explicar su Historia y para dar a la predicción un cometido propio de Ciencia Aplicada de Diseño. Esto comporta anticipar el futuro posible, para prescribir cómo actuar ante problemas concretos (SIMON, 1990). Pero, en este caso, hay que resolver los problemas derivados de propiedades emergentes con características bien distintas a las anteriormente conocidas (como sucede con frecuencia en las novedades introducidas por Internet).
Por un lado, para explicar la trayectoria del fenómeno de la complejidad dinámica de Internet, no cabe meramente concebirlo como evolución. Así, frente a lo que se repite con frecuencia (YIN et. al 2014, p. 14-19, HAN, 2012), este vigoroso crecimiento de Internet no es, sin más, una evolución, pues su complejidad es genuina historicidad (como se aprecia en el campo de la Comunicación). Por otro lado, para predecir su trayectoria futura - que tiene una inequívoca repercusión social, relacionada con la gestión pública y los sistemas multiagentes (multi-agents) (FLORIDI, 2014, p. 167-204)-, hay que afrontar la cuestión de las propiedades emergentes (NICOLIS; NICOLIS, 2012, MERALI, 2016), que no son meras "mutaciones" sino normalmente fruto de diseños humanos que introducen variaciones apreciables (como sucedió con el correo electrónico).

Esta doble tarea acerca de la complejidad dinámica de Internet se puede realizar desde las Ciencias de Diseño. Primero está explicar cómo se conforma esa complejidad dinámica en Internet, lo cual requiere verla desde la perspectiva de su configuración científica; y, después, se requiere predicción del futuro posible, para prescribir pautas de acción adecuadas para resolver los problemas de esa complejidad dinámica. Esta segunda vertiente nos lleva a las Ciencias Aplicadas de Diseño, que profundizan en la relación entre predecir y prescribir, un nexo que, en principio, precede a la aplicación de la Ciencia, que es la tarea realizada por los usuarios de la Red que utilizan sus conocimientos científicos en contextos delimitados (p. ej., farmacológicos o educativos).

Incide esa doble faceta de complejidad configuración y predicción - en la infraestructura de la Tecnología, sobre la que se asienta el contenido de la Red. Pero la plataforma tecnológica no es suficiente, por sí sola, para dar razón de la complejidad de Internet. Porque las Tecnologías de la Información y la Comunicación tienen dos funciones principales: a) son el soporte que hace viable la existencia de lo accesible en la Red; y b) condicionan la configuración de los contenidos de la Red, de modo que la dinámica depende de la manera de articular la infraestructura tecnológica "que tiene sus problemas, derivados asimismo de su creciente complejidad, lo que genera puntos de vulnerabilidad y dificultad para su manejo (YUAN; GONG 2011, p. 424)". Esta 
infraestructura propicia la complejidad dinámica de la Red, cuando conforman la base sistemas de información y comunicación con las siguientes propiedades emergentes: 1) que se pueda compartir, 2) esté abierto, 3) dé lugar a un conjunto heterogéneo de elementos y 4) que pueda cambiar con el paso del tiempo (HANSETH; LYYTINEN, 2010, p. 3).

Concebida como infraestructura para la complejidad dinámica - en cuanto plataforma tecnológica multivariada - , Internet tiene una relación bidireccional en el ámbito de la investigación científica aplicada. I) Internet es un soporte tecnológico que permite y hace posible el desarrollo de Ciencias Aplicadas de Diseño, como es el caso de las Ciencias de la Comunicación (en cualquiera de las formas de comunicación digital, ya intervenga texto, imagen o sonido), que ha ampliado la capacidad de resolución de problemas concretos y ha abierto un dilatado campo para nuevos objetivos, procesos novedosos y resultados innovadores). II) El avance de las Ciencias Aplicadas de Diseño (como las Ciencias de la Comunicación, pero también las Ciencias de la Documentación, la Educación en línea, etc.) impulsa nuevos desarrollos en Internet como Red de redes, pues obliga a la creación de nuevos programas informáticos que requieren soportes tecnológicos más sofisticados.

\section{MODALIDADES DE CIENCIA RELACIONADAS CON INTERNET}

Como el problema de la complejidad dinámica en Internet se afronta aquí desde un ángulo científico, hay que considerar las relaciones entre Internet y la Ciencia. A tal efecto, se consideran aquí tres aspectos: 1) las Ciencias actualmente existentes relacionadas con Internet desde la vertiente interna, 2) las modalidades de investigación científica relacionadas con la Red en el momento actual, y 3) las perspectivas endógena y exógena acerca de Internet.

\section{I Ciencias actualmente existentes relacionadas con internet}

Actualmente, la Ciencia de las Redes (network science o science of networks), la Ciencia de la Web (web science), la Ciencia de Internet en sentido estricto (Internet science), y la Ciencia de Datos (data science) - que puede ser transdisciplinar: (CAO, 2017, p. 60) - son las principales disciplinas científicas directamente relacionadas con Internet. Configuran de facto un marco interdisciplinar: la Ciencias de Internet, siendo cada una de las Ciencias participantes una disciplina interdisciplinar (TIROPANIS et al., 2015). Progresivamente propician el llegar a conformar un campo transdisciplinar de Ciencias de Internet, donde, además de las Ciencias de Diseño, tengan un papel claro las Ciencias Sociales.

Ciencia de las Redes es una denominación que tiene raíces en la Matemática, pues el estudio de las redes abarca un amplio campo: "incluye desarrollar modelos explicativos para captar las propiedades emergentes (emergence) de las redes, construir modelos predictivos para anticipar la evolución de las redes, y elaborar modelos prescriptivos para optimizar los resultados de las redes" (TIROPANIS et al., 2015, p. 76). Mientras tanto la Ciencia de la Web se ocupa de la creatividad de los agentes que intervienen en la Red utilizando la intermediación de las máquinas, con variaciones en el nivel micro (pequeños cambios en los diseños) o macro (que afectan globalmente a actividades societarias y comerciales) (TIROPANIS et. al., 2015, p. 78). Aquí la interacción interno-externo es patente, toda vez que la web es un fenómeno social con consecuencias para el propio desarrollo de la Red.

Ahora bien, la propia plataforma tecnológica de la Red necesita la aportación de la Ciencia, tarea a la que contribuye la Ciencia de Internet en sentido estricto, que ha contribuido al éxito de Internet, frecuentemente atribuido a su "modelo de gobierno distribuido, el principio de neutralidad de la Red y su apertura" (TIROPANIS et al., 2015, p. 78). Acerca del contenido se ocupa la Ciencia de Datos (data science), que ha de afrontar el tratamiento del gran volumen de datos (big data). A partir de un diseño de Internet en esos términos, se ha planteado el futuro desarrollo de la Red (YIN et al., 2014, p. 14-16), que hasta ahora se ha centrado en "conceptos y temas de Estadística, minería de datos (data mining), aprendizaje de máquina, y análisis de datos de amplio espectro" (CAO, 2017a, p. 59).

Rasgo común a todas estas disciplinas es la relevancia que conceden a la predicción. Este aspecto se resalta en la Ciencia de Datos, donde 
hay analítica predictiva (predictive analystics) (CAO, 2017b, p. 4). La predicción influye en modelos descriptivos acerca del futuro posible, pero también repercute en modelos prescriptivos sobre cómo debería ser el futuro buscado. Esas predicciones pueden ser acerca de las redes, sobre el propio desarrollo de la web y la contribución científica a la innovación tecnológica en la plataforma de Internet. Además hay predicciones basadas en datos masivos (big data), que requieren uso otros fines científicos, como son los relacionados con las Ciencias de la Comunicación (tanto para su desarrollo como Ciencia de lo Artificial como para estudiar la repercusión social de sus avances comunicativos, como la televisión por Internet).

\subsection{Tres modalidades de investigación científica relacionadas con la red}

Son al menos tres las modalidades actualmente existentes de actividad científica que, relacionadas con la Red, inciden en el problema de la complejidad dinámica. La primera modalidad lo hace de modo directo al afectar de lleno al propio desarrollo de la Red como tal - , sabiendo que el desarrollo de Internet es, con frecuencia, no lineal. Pero la no linealidad también se da en los sistemas naturales y sociales (BERTUGLIA; VAIO, 2005). La segunda repercute de manera indirecta, en cuanto que atañe a los usos de Internet para nuevas formas de hacer Ciencia. Y la tercera puede considerarse como Ciencia de Ciencia, en un sentido análogo a cómo las Ciencias de la Documentación son una actividad científica que presupone otras actividades científicas.

Está, en primer lugar, Internet como desarrollo de formas de Ciencia de lo Artificial. Se despliega mediante Ciencias de Diseño como la Ciencia de la Web o la Ciencia de Internet en sentido estricto-, en cuanto que sus objetivos, procesos y resultados son fruto de diseños pensados para ampliar las posibilidades humanas, sobre todo de comunicación e intercambio de información. Estas tareas iniciales de Internet acompañan ahora a otras tareas progresivamente más complejas, tanto en términos estructurales como dinámicos "como hacen, de modo habitual, empresas como Google, Facebook, Apple, Samsung, etc." (AUTOR 2017).
Supone esto que podemos hacer teorías y modelos sobre fenómenos nuevos generados por el desarrollo de Internet, en general, y la web, en particular, en cuanto que potencian las capacidades humanas. En este despliegue de Internet en clave científica, principalmente a través de la creatividad en la web, juega un papel clave la Inteligencia Artificial - que Simon (1995) consideraba Ciencia Empírica, de modo que los resultados se pueden controlar mediante pruebas que no son formales-, que interactúa con la innovación tecnológica de modo habitual.

Cabe, en segundo término, considerar Internet como nuevo saber instrumental, por su contribución para poder desarrollar nuevas formas de Ciencia - como son la Ciencia de Datos (science data) o a la Ciencia de las Redes (science of networks), que ciertamente considera aspectos estructurales y dinámicos (NEWMAN; BARABÁSI; WATTS, 2006)-, que amplían nuestro conocimiento por rutas distintas a las tradicionales. Porque el soporte tecnológico de Internet, como red multivariada, permite utilizar la Red como base para extraer datos relevantes y redes de relación, al modo cómo se trabaja en un laboratorio para alcanzar pautas (económicas, comunicativas, etc.) de interés científico en observaciones y experimentos.

Ofrece conocimiento del mundo natural, social o artificial a partir de lo obtenido en el entorno virtual. Permite, a tenor de los datos o de las redes de relaciones, apreciar propiedades de la Naturaleza, conductas sociales o aportaciones artificiales. También Internet - en colaboración con la Inteligencia Artificial - propicia el hacer pruebas de Ciencias de lo Artificial, puesto que -en interacción con agentes humanospodemos seguir investigando sobre contenidos diseñados (textos, imágenes, sonidos, gráficos, etc.), haciendo hipótesis que tienen interés científico y contrastándolas (como ha hecho Google Translate, que no puede reemplazar a los humanos, pero que resulta extremadamente útil).

También, en tercera instancia, cabe considerar a la Red como un nuevo "mundo" - el ciberespacio o la infoesfera -, distinto del mundo natural y diferente del social, donde aparecen nuevos fenómenos o eventos, precisamente por las características de Internet. Así, este tipo de Ciencia no mira al desarrollo mismo de la Red - en cuanto potenciadora de posibilidades humanas de información y comunicación - o 
busca hacer investigación que utilice Internet de modo instrumental (como hace la Ciencia de Datos, para ver pautas que expresen información médica relevante de un país), sino que sería un estudio empírico de los fenómenos que han surgido a raíz de Internet y que antes no podían darse.

Aquí, en este tercer tipo de Ciencia de Internet, se da una cierta "reificación": lo inicialmente virtual cobra forma con un nuevo tipo de efectos, que son semejantes a los que se pueden dar en la relación de los agentes humanos con la Naturaleza o en las dinámicas sociales como ciudadanos. Una investigación de este tipo es, por ejemplo, la realizada por los gestores económicos, que "necesitan de manera creciente entender cómo los consumidores usan e interactúan con los medios digitales, tales como la web, las redes sociales, los teléfonos móviles, los lectores digitales, los motores de búsqueda y así sucesivamente" (PAGE; UNCLES, 2014, p. 2356). Porque este tipo de estudio tiene, para ellos, muchas consecuencias prácticas con efectos económicos y sociales.

\subsection{Perspectivas endógena y exógena acerca de internet}

Cada una de las tres opciones científicas mencionadas se puede analizar a partir de dos grandes perspectivas: la endógena y la exógena. Ambas están directamente relacionadas con la actividad de investigación desarrollada de la Red y en sintonía con lo señalado acerca de las dos vertientes - la interna y la externa - de la diversificación de la Red. Estas perspectivas inciden en la complejidad dinámica de Internet en la triple dimensión considerada (en cuanto tal, en su utilización instrumental para nuevos tipos de estudios y como generadora de nuevos fenómenos empíricos). La vertiente externa puede incidir $-\mathrm{y}$, de hecho, lo hace- en la vertiente interna de la Red (YOO, 2012). Esto supone que se "internalizan" nuevos objetivos en Internet a tenor de una demanda "exterior", que puede estar debida a nuevas posibilidades tecnológicas, iniciativas de los usuarios, necesidades suscitadas por nuevos tipos de negocio, etc.

I) Mediante la perspectiva endógena, Internet aparece como una plataforma multivariada para desarrollar Ciencia de lo
Artificial, que busca dar soluciones a problemas concretos a través de nuevos diseños que amplían lo humano, bien sea de una manera "horizontal" (la ampliación de territorio, como la radio digital o la televisión digital) o bien de una forma "vertical" (la creación de nuevas formas comunicativas, como el correo electrónico, las redes sociales o sistemas interactivos de comunicación audiovisual tipo YouTube). En todos estos casos hay una interacción entre creatividad científica e innovación tecnológica (AUTOR, 2013b).

A su vez, la perspectiva endógena se puede desglosar en dos opciones principales: a) el desarrollo de esa Ciencia de lo Artificial como tal, esto es, como una actividad que busca expresamente resolver problemas científicos en un dominio nuevo (el mundo virtual o infoesfera); y b) la utilización de esa Ciencia de lo Artificial con carácter instrumental, esto es, para que esa Ciencia Aplicada de Diseño sirva para propiciar nuevos contenidos de otra índole: interdisciplinares, multidisciplinares o transdisciplinares.

II) Desde la perspectiva exógena, cabe hacer una Ciencia basada en Internet, donde la Red se use como laboratorio o sede de pruebas controladas. Esto propicia un nuevo tipo de investigación, en un entorno distinto al físico o al propiamente social, pues se ocupa de fenómenos que se dan a partir del uso del ciberespacio o esfera del mundo virtual. Esta Ciencia, apoyada en el comportamiento de los usuarios de la Red (individuos, grupos u organizaciones, públicas o privadas), puede ser de utilidad tanto para las Ciencias Sociales como para las Ciencias de lo Artificial.

Fenómenos nuevos, principalmente comunicativos, los generan las redes sociales (Facebook, Google+, Twitter, LinkedIn, Academia.edu, Research Gate, etc.). Lo hacen como sociedades artificiales, que pueden servir de punto de contraste respecto de otros fenómenos sociales de carácter rural o urbano. También en la Red hay diseños específicos para difundir noticias, que son de muy diversa índole (económica, política, cultural, etc.) y que inciden en la toma de decisiones de los agentes conectados a la web (en la que cabe la posibilidad de una futura sectorialización de la Red, esto es, que se formen ramas especializadas de la Red, tales como Internet industrial, Internet científica, 
Internet educativa, etc.). Así, se pueden estudiar fenómenos comunicativos mediante los Juegos de Realidad Alternativa o cabe indagar procesos de toma de decisiones económicas considerando el caso del uso de Bitcoins (una moneda generada en la Red).

\section{EL PAPEL DE LA PREDICCIÓN}

Tiene la predicción un papel clave en las tres modalidades de investigación relacionadas con la Red: a) en las Ciencias directamente enfocadas hacia Internet, pues puede versar sobre contenidos, funciones o aplicaciones de la Red como tal (p. ej., en la Ciencia de la Web o en la Ciencia de Internet en sentido estricto), b) cuando se realiza en Internet una actividad científica de tipo instrumental (p. ej., al desarrollar la Ciencia de Datos al servicio de la Medicina), y c) cuando Internet se utiliza como laboratorio, como sucede en disciplinas científicas concretas (como las Ciencias de la Comunicación), que usan la Red para fines específicos. Todos estos casos tienen repercusión en términos de complejidad dinámica (AUTOR; COLEGA, 2015, p. 304-309).

Es clave la predicción porque, primero, hay que anticipar el futuro posible - de la propia Red, de su uso instrumental o de los resultados del laboratorio - , para poder guiar la ampliación o potenciación de las posibilidades humanas, que repercuten en la vida social (Internet incide hoy en prácticamente en cualquier aspecto de vida). Después, tras la predicción viene la prescripción, el establecimiento de pautas encaminadas a la resolución de problemas concretos en un número finito de pasos. Porque la Ciencia Aplicada mira su acierto en la resolución de problemas concretos (NIINILUOTO, 1993), dentro de un dominio práctico (sea la propia Internet, su uso instrumental o su utilización en terrenos sociales, culturales, etc.), de modo que necesita anticipar lo posible, para luego guiar la acción (AUTOR, 2015).

\section{I Tres facetas sucesivas: objetivos, procesos y resultados}

Prima facie, la predicción en las Ciencias de Diseño - como las Ciencias de Internetpuede tener, en principio, tres facetas sucesivas: a) puede ser un objetivo destacado de las teorías vinculadas a diseños, que necesitan las predicciones acerca de lo viable antes de plantear prescripciones para cómo actuar; b) la predicción comporta procesos - procedimientos y métodos - para hacer avanzar el conocimiento científico, donde pueden intervenir factores teóricos, empíricos y heurísticos, que sirven de antesala a las pautas de actuación para resolver problemas concretos; y c) puede servir como criterio de evaluación de los enunciados científicos propuestos, cuyo contenido - teórico, empírico y heurístico - ha de ser utilizado en los criterios prácticos de resolución de problemas en liza como en el caso de (TURING; HODGES, 2014). Cada una de estas facetas sucesivas requiere atención.

1) Si el cometido se centra en el plano de los objetivos de los diseños, entonces intervienen tres tipos de predicciones. Primero, predicción epistemológica, pues hace falta un enunciado cuyo contenido cognitivo se considere correcto, bien sea en términos de previsión, de predicción en sentido estricto o de pronóstico (AUTOR, 2015, p. 68-72). Este enunciado establece la viabilidad de la meta en términos de racionalidad. Segundo, predicción de índole metodológica, que versa sobre los métodos posibles para resolver nuevos problemas estimados a tenor de la predicción de contenido. Porque todo método trabaja en conexión con un objeto real o posible, alcanzable en un número finito de pasos. Tercero, predicción contextual, que anticipe los elementos de entorno que pueden influir en la viabilidad de los diseños. Porque como son ampliaciones de las capacidades humanas, han de ser asumidas por el entorno (p. ej., en Economía Financiera, en los nuevos diseños en Documentación o en las nuevas formas de Comunicación).

2) Cuando la atención se centra en los procesos, entonces cabe repensar algunas propuestas filosófico-metodológicas que, para la predicción en Ciencias Empíricas, hizo Imre Lakatos acerca de su contenido (teórico, empírico y heurístico) (LAKATOS; ZAHAR, 1976 [1978], p. 179; LAKATOS, 1970 [1978] p. 33-34). Se pueden reconsiderar sus propuestas utilizándolas para el análisis de las Ciencias de lo Artificial, en general, y las Ciencias de Diseño, en particular, un ámbito que quedó fuera de su foco temático. Esto se traduciría en los siguientes rasgos sobre las predicciones científicas en cuanto procesos: 
(i) Con las predicciones puede haber progreso teórico o de avance en los modelos teóricos o en la aportación conceptual de las teorías, como sucede en las versiones de Inteligencia Artificial de redes neuronales utilizadas por Google, que llegan más lejos que los modelos computacionales de GOFAI tipo Simon. Esto es lo que sucede con el llamado "Cat paper" de los investigadores de Google (LE et al., 2012). (ii) Mediante las predicciones se da avance empírico: cabe el incremento de apoyo empírico respecto de predicciones anteriores, que se puede apreciar en el nivel de corroboración de las mediciones (como acaece en el aprendizaje de máquina de las últimas décadas, que contribuye a la Ciencia de Datos). (iii) Mejora heurística, que lleva a nuevas pautas de búsqueda para la resolución de problemas (como se constata, por ejemplo, en modelos econométricos cuando se analizan una serie de alternativas para anticipar el futuro posible a partir del conocimiento actualmente disponible).

3) Incide lo anterior en la evaluación de las predicciones. Porque estas tres funciones -teórica, empírica y heurística- se pueden desarrollar a tenor del grado de control de las variables disponibles. Esto atañe a la distinción entre previsión, predicción y pronóstico. Son diferentes de planeamiento o planificación, que es un concepto distinto, al estar encaminado hacia la dirección de la acción, en razón de cálculo y distribución de tiempos. Este enfoque, ya desarrollado para la Economía (AUTOR, 2015, p. 317-338), se puede utilizar para Internet en cuanto vía de desarrollo de las Ciencias de la Comunicación. Además de ser Ciencias Sociales, son Ciencias de lo Artificial, como se aprecia en los fenómenos de televisión digital (AUTOR, 2008b) o la televisión por medio de Internet conocida como Over The Top. Asimismo, mediante el estudio de la comunicación en Internet, se constata que son Ciencias Aplicadas de Diseño (AUTOR, 2012).

\subsection{Control de variable: previsión, predicción en sentido estricto y pronóstico}

Sucede que la evaluación de las predicciones relacionadas con el futuro de las Ciencias de Internet, en cuanto que Ciencias de Diseño, depende del tipo de enunciación predictiva (previsión, predicción en sentido estricto y pronóstico), que se apoya especialmente en el grado de control de la variable considerada. Aquí caben las tres posibilidades señaladas:

I) Se puede dar una anticipación en términos de previsión, que es cuando el grado de control de la variable es claro. Así, podemos hacer una previsión de un objetivo que sabemos es claramente alcanzable sobre la base del conocimiento actual (como, p. ej., lo que intenta hacer Facebook con el uso de drones en África para hacer accesible Internet en las zonas sin líneas telefónicas).

II) Cabe una anticipación del futuro comunicativo posible en Internet en términos de predicción en sentido estricto. Esto supone que hay elementos que controlamos y otros que no, como en la predicción sobre el uso de YouTube como soporte informativo en el medio y largo plazo. Así, el contenido transmitido vía YouTube puede estar más o menos perfilado, en términos de imagen, texto y sonido. Pero hay siempre un componente contextual que modula la comprensión, la interpretación y la aplicación de ese contenido informativo. Este componente contextual tiene elementos culturales, sociales, históricos, políticos, etc., que pueden condicionar la imagen, el texto y el sonido recibido.

III) Pronóstico es la anticipación del futuro posible que trae consigo el reconocimiento de un margen de error. Así, el futuro predicho se sabe que contiene, de entrada, algo de error. Esto se puede afirmar de los nuevos desarrollos de Google Translate, que aun cuando ha mejorado en los últimos años (LEWIS-KRAUS, 2016, p. 7-18), comporta un margen de error, por diversas razones: la existencia de palabras con un doble sentido, los contextos de uso de los términos utilizados, los modismos que son característicos de una zona cultural o de un país concreto, etc.

Planeamiento o planificación comporta, en efecto, anticipación del futuro posible sobre la base del conocimiento actual (un tipo de predicción en alguno de los sentidos mencionados: previsión, predicción estricta y pronóstico). Pero, además, planeamiento comporta la dirección de la acción, para lo cual requiere cálculo y distribución en el tiempo (plazo inminente, corto, medio, largo y muy largo plazo). Así, los planes de futuro de Twitter o de Snapchat pueden ser concebidos en términos de planeamiento o planificación. 


\section{EL PROBLEMA DE LA COMPLEJIDAD EN INTERNET EN CLAVE DE HISTORICIDAD}

Cuando se atiende a las Ciencias de Internet y su incidencia en las Ciencias de la Comunicación, cabe considerar las dos vertientes principales de complejidad: la estructural o de configuración y la dinámica o de cambio. Se aprecia que la complejidad estructural de los fenómenos comunicativos y de intercambio de información en Internet depende frecuentemente de desarrollos de Inteligencia Artificial. Así, empresas como Google, mediante el desarrollo de diversos proyectos, ha sido capaz de mostrar creatividad en el aprendizaje de máquina basado en redes neuronales, que ha llevado a avances en Google Translate y en el reconocimiento facial "p. ej., a través de algoritmos de reconocimiento de imágenes de gatos" (LEWIS-KRAUS, 2016, p. 7-18).

Comporta esto que las predicciones sobre futuros avances comunicativos en Internet (la posibilidad de nuevos diseños comunicativos que amplíen las demandas comunicativas de los usuarios particulares y las organizaciones) pasan, en principio, por predicciones respecto del conocimiento de nuevas formas de programación en Inteligencia Artificial, bien sea en términos computacionales, conexionistas o de sistemas de información de nueva índole (FLORIDI, 2011). A su vez, esas actividades científicas de desarrollo de Internet requieren el soporte tecnológico adecuado y el apoyo de instituciones - públicas o privadas - capaces de respaldar esas nuevas formas de comunicación y su consiguiente expresión en términos de Ontología de lo artificial.

Pero esos avances comunicativos vía Internet requieren afrontar la predicción de la complejidad dinámica, que no se detiene en procesos, sin más, y que no se queda tampoco en fenómenos evolutivos - tanto los meramente adaptativos como los que incluyen mutaciones con los cambios-, porque hay que llegar a la complejidad de Internet en términos de historicidad.

1) Historicidad (Geschichtlichkeit/historicity) es un rasgo de la Ciencia, en general, y de cada Ciencia, en particular, incluyendo a las Ciencias de Diseño. Esta característica puede ser dual: a) se puede encontrar en los componentes "internos" de la Ciencia (tales como lenguaje, estructura, conocimiento, métodos, actividad, fines y valores); y b) aparece en las relaciones "externas" entre Ciencia y su entorno (social, cultural, político, económico, ecológico, etc.). En Internet, en cuanto desarrolla actividad científica, se han dado $-\mathrm{y}$ se siguen dando- variaciones en los componentes internos y cambios en las relaciones externas, hasta el punto de ser considerada con frecuencia una parte importante de la "revolución digital", que tiene, además, consecuencias sociales y económicas importantes (BRYNJOLFSSON; MCAFEE, 2011).

2) La historicidad configura a los agentes que hacen Ciencia de varias maneras: (i) se da en los agentes dedicados al desarrollo de la investigación científica, en la medida en que son seres humanos dentro de un contexto histórico (que varía doblemente: al cambiar de zona geográfica a otra y por los factores que configuran ese contexto, cuando pasa de un momento a otro); (ii) aparece en las relaciones humanas entre los agentes en los centros de investigación (laboratorios, departamentos, etc.); y (iii) se encuentra también en las relaciones entre los agentes y la realidad (natural, social o artificial).

3) También la historicidad es una característica de la realidad misma investigada, sobre todo, en el campo social y en el ámbito de lo artificial. A este respecto, como Red de redes, Internet pertenece primariamente al terreno artificial -que genera un mundo virtual-, donde la desarrollan agentes humanos, que hacen Ciencia con la idea clara de la posibilidad de cambios profundos, tanto a nivel conceptual como en la faceta práctica (los nuevos tipos de instrumentos) y en el espacio institucional $u$ organizativo.

Si el foco de la historicidad de Internet se pone en la complejidad dinámica, a partir de la idea de la Ciencia como actividad humana, donde el progreso científico está marcado por la racionalidad, entonces se aprecia mejor el cambio profundo dado en Internet como red clave para la comunicación actual. En efecto, la Red de redes, por ser una plataforma multivariada, que está abierta a la novedad en los tres grandes campos de la racionalidad: el plano cognitivo, la vertiente práctica y la dimensión evaluativa (RESCHER, 1988): 
a) Internet comporta cambios en el conocimiento adquirido o adquirible y en nuestro acceso a nuevas facetas artificiales, en cuanto que es medio y no fin. b) Internet potencia la toma de decisiones humanas al abrir nuevos espacios prácticos de índole virtual, no existentes antes en la Historia de la Humanidad. c) Internet es también vehículo para el cambio de valores, porque las redes sociales, en general, y el modus operandi de sociedades artificiales llevan a caracterizaciones nuevas del tipo de valores relevantes, al menos para un gran número de personas.

\section{I Complejidad dinámica cognitiva en términos de historicidad}

Junto a la novedad que comportan "giros históricos", que pueden conllevar cambios relevantes - como ha sucedido en cuanto al acceso y recuperación de la información vía Internet-, ha habido una auténtica revolución en cuanto a la interactividad para contenidos informativos, tanto en formato texto como de tipo audiovisual. En las últimas décadas, esto se ha logrado, además, en dos direcciones: la horizontal y la vertical.

Ha habido un progreso horizontal, en la medida en que se ha dilatado el modo de llegar al conocimiento sobre la base de medios antes analógicos - como la radio digital o la televisión digital - y ha tenido lugar un progreso vertical, en cuanto que hay novedades netas, tales como una superposición de niveles cognitivos, en virtud de algoritmos que permiten llegar con motores de búsqueda -Google, Yahoo, Bing, etc. - a contenidos informativos en la Red, vía hipertextos, metadatos, etc., El futuro posible pasa aquí, en principio, por la ruta de avances en Inteligencia Artificial para nuevos objetivos, que ha de dar lugar a nuevos procesos, que permitan llegar a resultados novedosos.

\subsection{Complejidad dinámica práctica concebida desde la historicidad}

Acontece que, de modo semejante a cómo el lenguaje -capacidad universal- se actualiza en entornos delimitados y da lugar a actos de habla - locucionarios, ilocucionarios y perlocucionarios-, Internet - plataforma multivariada - tiene una dimensión práctica de índole histórica mediante la web de esta Red de redes. Porque hacemos "actos de web", esto es, hacemos acciones en la web que tienen los tres tipos señalados: (i) unos actos poseen un carácter descriptivo - como los correos electrónicos-; (ii) hay otros actos en la web que comportan efectos formales o institucionales (p. ej., cuando rellenamos un cuestionario para una convocatoria de una plaza o damos la conformidad a una plataforma para visados, para poder viajar a un país concreto, etc.); y (iii) hay, finalmente, otros actos en la web que atañen a terceros, con consecuencias (p. ej., al hacer un informe de evaluación, al poner unas calificaciones en la web, al dar a conocer un accidente de tráfico que acaba de ocurrir, etc.).

De nuevo, estos tres tipos de actos de web revisten con frecuencia mayor complejidad práctica que actos comunicativos en la etapa analógica. Porque no hay únicamente una mayor dilatación del territorio accesible - la web puede ser vista por muchos, tanto los destinatarios como los hackers - como por las repercusiones en términos de inmediatez y consecuencias prácticas a diversos niveles (como cuando gobernantes, como el actual Presidente de Estados Unidos, comunican sus decisiones o apreciaciones vía Twitter).

\subsection{Complejidad dinámica evaluativa abierta a la historicidad}

No se limita la complejidad dinámica de Internet a la complejidad cognitiva y práctica, pues también interviene la complejidad relacionada con valores (como puede ser "sociedad abierta"). Porque hay una serie de valores asumidos, de manera implícita o explícita, cuando configuramos una identidad digital. Es la identidad transmitida vía redes sociales (como LinkedIn, Academia.edu, Google+, Research Gate, etc.) o la que se infiere a partir de las informaciones disponibles en la web. Esa identidad digital se elabora en razón de unos valores - según sea el tipo de red social: profesional, de amistad o relaciones, etc. - y cambia en razón de la modificación de los valores prioritarios para cada persona, para el grupo al que se desee conectarse o para la sociedad 
artificial que se genera en el ciberespacio o entorno digital en general.

Variar este tipo de valores prioritarios - normalmente, los dominantes en la actual Sociedad del Conocimiento - con lleva cambiar el tipo de comunicación elegida. Podemos ser "cosmopolitas domésticos" (ECHEVERRÍA, 1995), pero está condicionado históricamente: no se es cosmopolita digital en países sin libertades públicas, donde hay control político de Internet, mientras que sí se puede ser tal en países con seguridad jurídica. Con todo, la predicción del futuro posible depende de cambios en la sociedad, sobre todo si es abierta. Así, dependemos de revoluciones culturales y de guerras, que tienen incidencia vertical (una supresión de valores, sobre todo los relacionados con la libertad), y de otros cambios culturales, que tienen repercusión más horizontal (como la accesibilidad a la televisión por Internet).

\section{CONSIDERACIONES FINALES}

Afrontar el problema de la complejidad dinámica en Internet - un sistema abierto, con constante proliferación de nuevas expresiones, abiertas en abanico a cambios en las diversas direcciones - requiere distinguir y profundizar en los diversos aspectos que intervienen. Primero están la creatividad científica y la innovación tecnológica: una fuente de complejidad dinámica es la actividad científica y otra es el quehacer tecnológico. Segundo, hay elementos de diversificación de carácter interno y externo en liza, por los cuales Internet no funciona en modo monoplano. Las Ciencias de Internet son lo más opuesto a una proyección lineal del trabajo de programadores, fácilmente previsible en cuanto a su futuro, pues la dinámica interno-externo de la Red marca su historicidad. Tercero, los cambios en Internet solo se entienden si se superan los esquemas de Simon para las Ciencias de Diseño. Supone resaltar la complejidad dinámica y hacerlo desde la historicidad - en vez de la mera evolución -, donde el campo artificial no es mera hechura humana (human made), sino que hay toda la variedad de elementos señalada asociada a la historicidad.

Desempeña la predicción un papel clave respecto de la complejidad dinámica de Internet, que incide en toda una nueva variedad de formas de actividad científica. Porque la Red no trabaja solo con un tipo de Ciencia: la generadora su propio desarrollo, con la ayuda de Inteligencia Artificial. También desarrolla actividad científica mediante el cometido de saber instrumental. Propicia, además, nuevos tipos de fenómenos en el mundo virtual, pero con repercusión social, que requieren investigación científica. Sobre esta base, Internet ha puesto a la predicción científica en esos tres planos filosófico-metodológicos. Porque la predicción es central para afrontar el futuro posible de la Red, a partir de su uso en las diversas Ciencias relacionadas con Internet, y en las otras dos modalidades de investigación científica señaladas. Todas requieren saber anticipar el futuro posible, para prescribir las pautas de actuación adecuadas para resolver los problemas planteados.

El vigoroso crecimiento de Internet en las últimas décadas no se explica meramente por ser una plataforma tecnológica, sino porque parte de diseños que modulan su contenido a tenor de objetivos deliberadamente buscados. En este sentido, el avance de Internet se explica principalmente en cuanto Ciencia de Diseño. Esto se ve con mayor claridad al profundizar en el caso de las Ciencias de la Comunicación, que usa las Ciencias de Internet, tanto de modo instrumental como para hacer observaciones y experimentos de nuevos fenómenos. Aparecen entonces muchos más factores en liza, tanto estructurales como dinámicos, con sus formas de complejidad epistemológicas y ontológicas, pues la Comunicación utiliza Internet como Red de redes. Así, debido a su carácter multivariado, Internet permite las diversas opciones futuro de las Ciencias de Diseño, como son los planteamientos específicos, los interdisciplinares, los multidisciplinares y los transdisciplinares.

\section{Agradecimientos}

Este artículo se ha preparado en el Center for Philosophy of Natural and Social Sciences (London School of Economics). Forma parte del Proyecto de Investigación FFI2016-79728-P, financiado por el Ministerio español de Economía, Industrial y Competitividad (AEI). 
Artigo recebido em 0I/02/20I8 e aceito para publicação em 0I/03/20I8

\section{DYNAMIC COMPLEXITY ON THE INTERNET AS AN INFORMATION AND COMMUNICATION PLATFORM:} philosophical analysis from the perspective of design sciences and the role of prediction

ABSTRACT

Due to the intense development of the Internet, quantitative and qualitative, the problem of dynamic complexity on this network has been generated. Its constant growth as an open system, where new expressions proliferate - of content, functions, etc. — gives rise to new forms of complexity, which are in themselves dynamic. To address this problem, a philosophical analysis is made here from the perspective of design sciences and the role of prediction, but seeking to overcome Herbert Simon's approach. Because the problem of dynamic complexity on the Internet should be addressed from the historicity in the cognitive, practical and evaluative realms.

Keywords: $\quad$ Growth. Network. Artificial. Future. Historicity.

\section{REFERENCIAS}

BERNERS-LEE, Timothy. Weaving the Web. Londres: Texere Publishing, 1999.

BERTUGLIA, Cristoforo S.; VAIO, Franco. Nonlinearity, Chaos and Complexity: The Dynamics of Natural and Social Systems. Oxford: Oxford University Press, 2005.

BISHOP, Robert C., Metaphysical and Epistemological Issues in Complex Systems. En HoOKer, Cliff (ed). Philosophy of Complex Systems. Ámsterdam: Elsevier, 2011, pp.105-136.

BRYNJOLFSSON, Erik; MCAFEE, Andrew. Race Against Machine: how the Digital Revolution is Accelerating Innovation, Driving Productivity, and Irreversibly Transforming Employment and The Economy. Lexington, MA: Digital Frontier Press, 2011.

CAO, Longbing. Data Science: Challenges and Directions. Communications of ACM, v. 60, n. 8, 2017a, pp.59-68.

Data Science: A Comprehensive Overview. ACM Computing Surveys v. 50, n. 3, art. 43, 2017b, pp.1-42.

ECHEVERRÍA, Javier. Cosmopolitas domésticos. Barcelona: Anagrama, 1995.
FLORIDI, Luciano. Philosophy of Information. Oxford: Oxford University Press, 2011.

The Fourth Revolution - How the Infosphere is Reshaping Human Reality. Oxford: Oxford University Press, 2014.

GONZÁLEZ, Wenceslao J. Rationality and Prediction in the Sciences of the Artificial: Economics as a Design Science. En GALAVOTTI, Maria Carla, SCAZZIERI, Roberto y SUPPES, Patrick (eds), Reasoning, Rationality, and Probability, Stanford: CSLI Publications, 2008a, pp. 165-186.

La televisión interactiva y las Ciencias de lo Artificial. En ARROJO, María José, La configuración de la televisión interactiva: De las plataformas digitales a la TDT, A Coruña: Netbiblo, 2008b, pp. xi-xvii.

Las Ciencias de Diseño en cuanto Ciencias de la Complejidad: Análisis de la Economía, Documentación y Comunicación. En GONZÁLEZ, Wenceslao J. (ed), Las Ciencias de la Complejidad: Vertiente dinámica de las Ciencias de Diseño y sobriedad de factores, A Coruña: Netbiblo, 2012, pp. 7-30.

The Sciences of Design as Sciences of Complexity: The Dynamic Trait. En ANDERSEN, Hanne, DIEKS, Dennis, GONZÁLEZ, Wenceslao 
J., UEBEL, Thomas y WHEELER, Gregory (eds), New Challenges to Philosophy of Science, Dordrecht: Springer, 2013a, pp. 299-311.

The Roles of Scientific Creativity and Technological Innovation in the Context of Complexity of Science. En GONZÁLEZ, Wenceslao J. (ed), Creativity, Innovation, and Complexity in Science, A Coruña: Netbiblo, 2013b, pp. 11-40.

Philosophico-Methodological Analysis of Prediction and its Role in Economics, Dordrecht: Springer, 2015.

GONZÁLEZ, Wenceslao J. y ARROJO, María José. Diversity in Complexity in Communication Sciences: Epistemological and Ontological Analyses. En GENERALI, Dario (ed), Le radici della razionalità critica: Saperi, Pratiche, Teleologie, Milán-Udine: Mimesis, vol. I, 2015, pp. 297-312.

From Intelligence to Rationality of Minds and Machines in Contemporary Society: The Sciences of Design and the Role of Information. Minds and Machines, v. 27, n. 3, 2017, pp. 397424. DOI: $10.1007 /$ s11023-017-9439-0. Disponible en https://link.springer.com/article/10.1007/s11023017-9439-0 (acceso 6.8.2017)

GRAHAM, Gordon. The Internet: A Philosophical Inquiry. Londres: Routledge, 1999.

HAN, Dongsu S. Supporting Long Term Evolution in an Internet Architecture. Pittsburgh: Carnegie Mellon University, 2012. Disponible en: <http://reports-archive.adm. cs.cmu.edu/anon/2012/CMU-CS-12-144.pdf>. Acceso: 7 agos. 2017.

HANSETH, Ole; LYYTINEN, Kalle. Design Theory for Dynamic Complexity in Information Infrastructures: The Case of Building Internet. Journal of Information Technology v. 25, n. 1, 2010, pp. 1-19.

HODGES, Andrew. Alan Turing: The Enigma. Vintage Books/Random House: Londres, 2014.
HOOKER, Cliff. Conceptualising Reduction, Emergence and Self-Organisation in Complex Dynamical Systems. En HOOKer, Cliff (ed). Philosophy of Complex Systems. Ámsterdam: Elsevier, 2011, pp.195-222.

LAKATOS, Imre. Falsification and the Methodology of Scientific Research Programmes. En LAKATOS, Imre; MUSGRAVE, Alan (eds). Criticism and the Growth of Knowledge. Cambridge: Cambridge University Press, 1970, pp. 91-196. Compilado en LAKATOS, Imre. The Methodology of Scientific Research Programmes. Cambridge: Cambridge University Press, 1978, pp.8-101.

LAKATOS, Imre; ZAHAR, Elie G. Why did Copernicus's Programme supersede Ptolemy's? En WESTMAN, Robert S. (ed.). The Copernican Achievement. Los Ángeles: University of California Press, 1976, pp.354-383. Compilado en LAKATOS, Imre. The Methodology of Scientific Research Programmes. Cambridge: Cambridge University Press, 1978, pp.168-192.

LE, Quoc V. et al. Building High-level Features Using Large Scale Unsupervised Learning. Proceedings of the 29th International Conference on Machine Learning, Edinburgh, Escocia, 2012. Disponible en: <https://static. googleusercontent.com/media/research.google. com/es//pubs/archive/38115.pdf >. Acceso: 11 mar. 2016.

LEWIS-KRAUS, Gideon. The Great A.I. Awakening. The New York Times Magazine 14 December 2016, pp. 1-37. Disponible en: <https://www.nytimes.com/2016/12/14/ magazine/the-great-ai-awakening.html?_r=0>. Acceso: 12 mar. 2017.

MERALI, Yasmin. Complexity and Information Systems: The Emergent Domain. Journal of Information Technology v. 21, 2016, pp.216-228.

NEWMAN, Mark; BARABÁSI, Albert-Lásló; WATTS, Duncan J., The Structure and Dynamics of Networks, Princeton: Princeton University Press, 2006. 
NICOLIS, Gregorie; NICOLIS, Catherine. Foundations of Complex Systems: Emergence, Information and Prediction. NJ: World Scientific, River Edge, 2012.

NIINILUOTO, Ilkka. The Aim and Structure of Applied Research. Erkenntnis v. 38, 1993, pp.121.

PAGE, Kelly L.; UNCLES, Mark D. The Complexity of Surveying Web Participation. Journal of Business Research v. 67, 2014, pp.2356-2367.

RESCHER, Nicholas. Rationality: A Philosophical Inquiry into the Nature and the Rationale of Reason. Oxford: Clarendon Press, 1988.

Complexity: A Philosophical Overview.

New Brunswick, NJ: Transaction Publishers, 1998.

SIMON, Herbert A. The Architecture of Complexity. Proceedings of the American Philosophical Society, v. 106, n. 6, 1962, pp.467482.

The Impact of Electronic Communications on Organizations. En Wolff, Rolf (ed). Organizing Industrial Development. Berlín: Walter de Gruyter, 1986, pp.251-256.

Prediction and Prescription in Systems Modeling. Operations Research v. 38, 1990, pp.714.
SIMON, Herbert A. Artificial Intelligence: An Empirical Science. Artificial Intelligence v. 77, n. 1, (1995), pp.95-127.

The Sciences of the Artificial, $3^{\mathrm{a}}$ ed., Cambridge, MA: The MIT Press, 1996.

Economics as a Historical Science. Theoria v. 13, n. 32, 1998, pp. 241-260.

TIROPANIS, Thanassis et al. Network Science, Web Science, and Internet Science. Communications of ACM v. 58, n. 8, 2015, pp.7682.

UHLIG, Steve. On the Complexity of Internet Traffic Dynamics on Its Topology. Telecommunication Systems, v. 43, n. 3, 2010, pp. 167-180.

WINTER, Jenifer; ONO, Ryota (eds.). The Future Internet: Alternative Visions. Dordrecht: Springer, 2015.

YIN, Hao et al. Big data: Transforming the Design Philosophy of Future Internet. IEEE Network, v. 28, n. 4, (2014), pp. 14-19.

YOO, Christopher S. The Dynamic Internet: How Technology, Users, and Businesses are Transforming the Network. Washington: AEI Press, 2012.

YUAN, Ruixi; GONG, Weibo. On the Complexity and Manageability of Internet Infraestructure. Frontiers of Electrical and Electronic Engineering in China v. 6, n. 3, 2011, pp.424-428. 